\title{
A GLIMPSE OF THE GAMBIA
}

MAJOR G. O. COWAN, M.B., Ch.B., M.R.C.P., M.R.C.P.E., D.T.M.\&H. *

R.A.M.C.

British Military Hospital, Munster.

\section{Introduction \\ "Ex Africa semper aliquid novi" Pliny}

The Gambia is an appendix-shaped enclave within Senegal, in West Africa, and is some 200 miles long East to West but encloses only a few miles of rain forest and savannah on either bank of the great River Gambia. It is the smallest country in Africa in size and has a population of some 600,000. The economy depends heavily on a single crop, the groundnut, which is exported mainly as oil. The people, who are predominantly Muslim, are a mixture of several West African tribes-Wolof, Mandingo, Fula and Jola. The Gambia river mouth was explored by the Portuguese in the days of Henry the Navigator and English ships sailed up-river in search of gold in the time of Queen Elizabeth I. The Gambia river colony became a pawn in the prolonged colonial struggle between Britain and France, and after Waterloo became a British possession, justifiably earning the name of "White Man's Grave" because of malaria, yellow fever, typhoid, alcoholism and suicide. Independence finally came in 1965 and The Gambia is now a peaceful Republic within The Commonwealth. The author was seconded briefly in 1974 to the Medical Research Council Laboratories, Fajara, near the quaint bustling little waterfront capital city of Banjul.

\section{The prevalence of liver disease}

The commonest problems in adult medicine in The Gambia are presented by liver disease, and the most usual presentation is with ascites, hepatomegaly and ankle oedema, without jaundice. In most such cases liver biopsies will show macronodular (postnecrotic) cirrhosis. In a few cases with a short history, massive ascites, jaundice and dilated abdonimal wall veins, the histology is that of veno-occlusive disease. Many factors may combine in damaging liver cells and venules:-

(a) Protein deficiency. (b) Hepatitis viruses A and B. (c) Fungal toxins. Chromatographic analysis of cereals and groundnut paste on sale in the markets reveals significant amounts of aflatoxins and ochratoxins which, it has been suggested, suppress the immune response to virus hepatitis; this immune incompetence is aggravated by lack of dietäry protein and by chronic malaria.

(d) Pyrollizidines. These alkaloids are found in plants, for example, Senecio, Crotalaria spp. used in "bush tea" in Jamaica and may also be contaminants of cereal crops. They are probably present in some of the native herbal medicines in The Gambia, and may be responsible for acute veno-occlusive disease of the liver.

*Now Lieutenant-Colonel, British Military Hospital, Hong Kong. 
In The Gambia two factors not implicated are alcohol, as the people are faithful Muslims, and iron such as from Bantu beer-pots.

From the prevalence of hepatic cirrhosis it follows that the commonest tumour of adults is primary liver carcinoma, presenting as a huge hard multilobulated hepatic mass in a middle-aged African (Figure 1 depicts one such



Fig. 1. Gambian female with massive hepatoma (Note marks of dressing at biopsy site).

female) who is likely to die within a few weeks. Early diagnosis in tropical circumstances is notoriously difficult, although theoretically serum alpha-fetoprotein measurement might be helpful. Late presentation is the rule, and cytotoxic therapy not even palliative, as a small trial of intravenous 5 -fluorouracil showed.

In some cases of hepatomegaly and ascites, however, liver biopsy may reveal treatable conditions such as tuberculosis or brucellar granulomata, or schistosomiasis. Schistosoma mansoni is found in small foci in the southern coastal areas of The Gambia in fresh-water ponds and one young adult male presented, after a single exposure 3 months previously, with a tender enlarged liver, jaundice and Schistosoma mansoni ova in his faeces. Liver biopsy showed granulomata surrounding ova, but no fibrosis, and the patient responded well to niridazole, and should not develop schistosomal fibrosis unless he in reinfected. 
Another possibility is the hepatic fibrosis of congenital syphilis. One splendid village headman rejoicing in the title of The Alkalo of Jambanjeli presented with ascites, hepatosplenomegaly, ankle oedema and a "saddle nose". 'His Wasserman reaction was a $4+$ positive, and liver biopsy showed characteristic non-cirrhotic fibrosis. He was treated with penicillin and a diuretic and improved.

Hepatic amoebiasis is common in The Gambia and, as in many tropical areas, is predominantly a disease of adult males. Two cases in females therefore proved especially interesting. The first of these presented with tender soft hepatomegaly, jaundice and low-grade fever, but was not seriously ill. Her serum gave a strongly positive test $(1: 1000)$ for antibodies to Entamoeba histolytica by immunofluorescence. Under observation for 2 weeks her condition remained unchanged, so she was then treated with high-dose metronidazole with good effect. The second case was of a young woman who had just aborted a 5-month foetus, and was admitted stuporose in obvious hepatocellular failure. Her liver was uniformly enlarged and soft. Her condition improved with conventional therapy and over the next 2 weeks she developed a large tender hepatic mass, and a hectic fever. Aspiration of the mass revealed "anchovy sauce pus" containing abundant trophozoites of Entamoeba histolytica and also active Trichomonas hominis, both of which protozoa were then cultured from the fluid. She responded well to treatment with emetine and chloroquine. It therefore appeared that these were two cases of diffuse amoebic hepatitis, the concept of Doxiades (1964) which has been discounted by most other authorities. It has more recenly been suggested that invasive hepatic amoebiasis, in countries where Entamoeba histolytica is ubiquitous and the general population mostly carry antibodies to it, implies a breakdown of T-lymphocyte efficiency. In these two cases, and two others in males, however, it was possible to study lymphocyte transformation in response to pure amoebic antigen from axenic culture, and in all four cases this response was normal. It therefore remains to be explained why Entamoeba histolytica becomes invasive in this way.

\section{Other adult diseases}

Cardiac failure is a common problem in adult medicine in The Gambia, and the most usual cause is rheumatic valvular disease. Another interesting problem is acute puerperal cardiomyopathy. This is usually a young primigravida presenting in the second week after delivery in acute left and right-sided heart failure. Anaemia, malnutrition and vitamin deficiency do not appear to be implicated and, because of the usually moderately elevated diastolic blood pressure, it has been suggested that this is a variant of the toxaemia-gestosis syndrome. These patients recover quickly with digoxin and diuretics and may not suffer again in the next pregnancy. Pueperal cardiac decompensation may also be due to severe anaemia with haemoglobin usually less than $6 \mathrm{~g} / \mathrm{dl}$ and the blood film of combined iron and folic acid deficiency. Cardiac output is usually high, so that transfusion may result in fatal pulmonary oedema; on the other hand over-vigorous diuretic therapy may cause a fatal low-output state. Treatment should therefore depend on parenteral iron and folic acid, and, if the patient's condition is critical, exchange transfusion should be attempted. 
It was noticeable that most of the patients seen with severe typhoid fever who had often been ill for two or three weeks were young women presenting with toxaemia, severe anaemia, diarrhoea and hepatosplenomegaly who frequently gave a history of recent pregnancy or miscarriage. Their response to chloramphenicol was gratifying.

Young adult men and women was massive splenomegaly are most commonly suffering from "tropical splenomegaly syndrome". Serum levels of IgM are markedly elevated and although malarial parasites are absent from peripheral blood, the splenomegaly responds to long-term antimalarial therapy.

Among neurological problems, occlusive cerebral arterial disease appears to be commoner in men in the 4th and 5th decade of life than coronary artery disease. Hypertension or diabetes mellitus may be associated, but more often the blood pressure is normal but the electrocardiogram shows evidence of left ventricular hypertrophy. Paraplegia may result from trauma, spinal tuberculosis, schistosomal myelitis, or a form of spastic-ataxic myelopathy caused by excessive reliance on cassava tuber as the staple food, from which large amounts of thiocyanate are released by gastric acid: This condition is probably aggravated by cigarette smoking and one young male with this condition improved rapidly in hospital when fed with a rice-based diet and denied cigarettes. Sleeping sickness is not common in the coastal areas of The Gambia but the patient's face and demeanor are almost always classical, and the diagnosis is most easily confirmed by finding motile Tryponosoma gambiense on direct dark-ground microscopy of unstained neck lymph-node aspirate or cerebro-spinal fluid. Progress on treatment with courses of melarsropol is slow but satisfactory.

Filariasis may take unexpected forms, early infections in small boys sometimes presenting with large scrotal hydrocoeles. One male adult who presented with suprapubic pain and chyluria was suspected of Bancroftian filariasis but his blood and urine contained the microfilariae of Dipetalonema perstans. This worm was once regarded as non-pathogenic but the adult forms are found in the retroperitoneal tissue, and in this case periureteric fibrosis and hydronephrosis were confirmed by excretion urography. Unfortunately this parasite does not respond to currently available filaricides.

\section{Malnutrition in children}

Protein-caloric malnutrition in all its well-described guises is common in The Gambia, most of these young weanling children presenting in a very weak condition after a long journey on their mothers' backs from the bush villages where cassava is the usual weanling diet, containing carbohydrate but very little protein. In the child welfare clinics near the capital city this problem is seldom seen, and it is quite usual to see grossly overweight chesty infants wheezing between gulps at the mothers' capacious breasts, and also fed with bottled milk, that dangerous and unnecessary badge of relative prosperity, which too often carries unpleasant strains of Escherichia coli and other pathogens. 
The pathogenesis of kwashiorkor can be summarised in the "Vicious Circle" in Figure 2, from which these children will be very lucky to escape without inten-

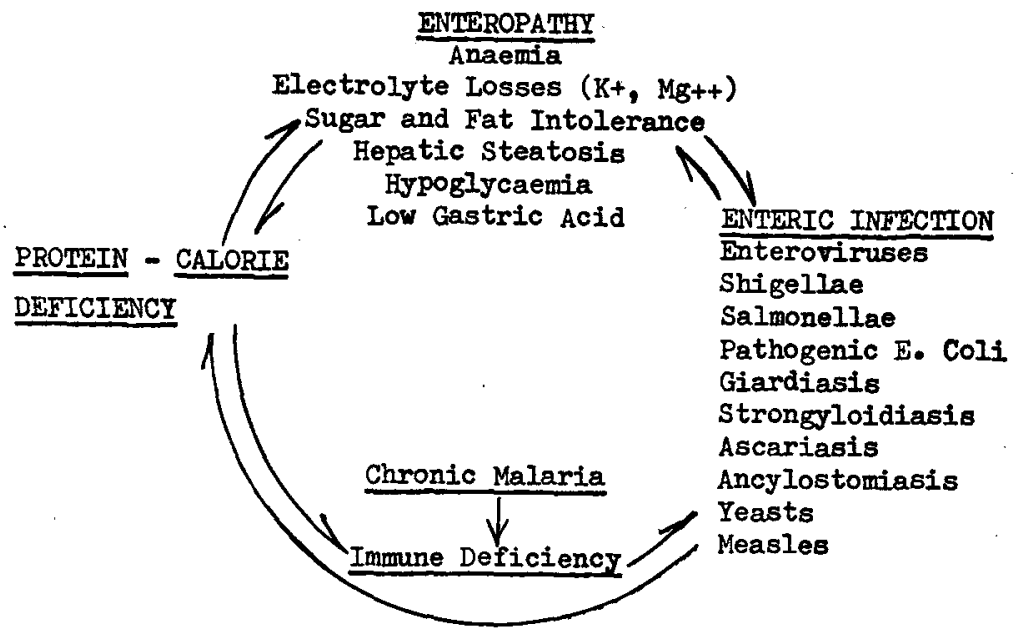

Fig. 2. The "vicious circle' 'of kwashiorker.

sive treatment. These children, usually aged 18 to 36 months, are apparently dehydrated because of muscle wasting but have in fact raised total body water and tissue oedema, low serum albumin and low circulating plasma volume. It follows that both intravenous fluids and diuretics can be dangerous, so that treatment should depend on very frequent very small feeds through a nasogastric tube of a mixture low in salt and high in protein and calories, usually vegetable oil, skim milk and sugar with added potassium chloride, magnesium hydroxide, iron, folic acid and B group vitamins. Stool and blood cultures are important as salmonellar and shigellar scepticaemia is common. Hypoglycaemia is common initially but intravenous dextrose aggravates hypokalaemia and corticosteroids are also unhelpful. Intestinal parasites, such as Giardia lamblia, Ascaris lumbricoides, should be appropriately treated. Hypothermia is most easily avoided by admitting mother and child to the same bed. Children who survive the first few days will usually recover. If the stools become frothy and acid, the sugar content of feeds should be reduced; if they become fatty, pancreatic extract should be added to the feeding mixture. Malaria may complicate the recovery phase as the red blood cell pabulum becomes more favourable for the plasmodia. Nor should tuberculosis be forgotten: an initial tuberculin test is always negative due to depressed immunity, and a "late-positive" result may be evident 2 to 3 weeks later as nutrition improves.

\section{Malaria, viruses and tumours}

Plasmodium falciparum malaria is hyperendemic in the Gambian countryside, the commonest presentation being a comatose fitting febrile child of nine months 
to four years. In the smaller children cerebral oedema is only too evident from the bulging anterior fontanelle and the author believes that treatment with intramuscular dexamethasone $(4 \mathrm{mg}$ ) is mandatory. A single intramuscular dose of chloroquine $(7 \mathrm{mg} / \mathrm{kg})$ is sufficient even with heavy infections, and the child may be found enjoying rice "chop" the next morning and can be discharged later that day. Survivors will have progressively less severe illnesses with new infections as immunity develops, so that attempts at chemoprophylaxis in this setting are almost certainly actively harmful.

The relationship of hyperendemic malaria to the incidence of Burkitt's lymphoma in children is now well established, and this tumour is fairly common in the five to 10 year age group. The relationship of the lymphoma to the EpsteinBarr virus has also been established. It has been postulated that in glandular fever, the abnormal circulating lymphocytes are T-forms which dispose of virusbearing B-forms. In children in whom chronic malaria has depressed T-lymphocyte function, the infected B-lymphocytes survive and multiply as lymphoma. Gambian children of the same age as the lymphoma victims are seen, but from more prosperous homes in the city where malarial transmission is low, with typical glandular fever which subsides normally. The source of the Epstein-Barr virus is not known for certain but the author suggests that it may frequently be carried by mosquitoes, partly because he bellieves that he acquired glandular fever himself in this way in The Gambia. Another much less common lymphatic tumour of childhood, of which one example was seen, is Kaposi's sarcoma, in which infection with Herpesvirus hominis in an immune-deficient setting has been postulated. Cytotoxic drugs such as cyclophosphamide may be remarkably effective in treating both tumours but care should be taken not to use excessive or too frequent dosage as this will depress the natural immune response to the tumour even further.

\section{Acknowledgements}

The author is most grateful to Dr. R. S. Bray and the staff of Medical Research Councill Laboratories, Fajara, The Gambia for their assistance to him, and to the Medical Superintendent and staff of The Royal Victoria Hospital Banjul for their invitation to him to act as Visiting Physician.

\section{REFERENCE}

Doxiades, T. (1964) J. Amer med. Ass. 187, 179.

\section{Queen's Honorary Physician}

BRIGADIER A. T. COOK, M.B., B.S., F.R.C.P., F.R.C.P.(Ed.), Late Royal Army Medical Corps, was appointed Honorary Physician to The Queen on 18 October 1977, in succession to Major-General W. O'Brien, who has retired. 